\title{
SIRT3 is correlated with the malignancy of non-small cell lung cancer
}

\author{
YANLU XIONG $^{1 *}$, MINGXING WANG $^{1 *}$, JINBO ZHAO $^{1 *}$, LEI WANG $^{2}$, \\ XIAOFEI LI ${ }^{1}$, ZHIPEI ZHANG ${ }^{1}$, LINTAO JIA ${ }^{2}$ and YONG HAN ${ }^{1}$ \\ ${ }^{1}$ Department of Thoracic Surgery, Tangdu Hospital, Fourth Military Medical University, Xi'an, Shaanxi 710038; \\ ${ }^{2}$ State Key Laboratory of Cancer Biology, Department of Biochemistry and Molecular Biology, \\ Fourth Military Medical University, Xi'an, Shaanxi 710032, P.R. China
}

Received December 1, 2016; Accepted January 30, 2017

DOI: $10.3892 /$ ijo.2017.3868

\begin{abstract}
The mitochondrial deacetylase SIRT3 plays a pivotal role in the initiation and the progression of certain cancers acting as an oncogene. However, in others it acts anti-oncogenically. Its conflicting action is possibly due to the different key proteins it modifies depending on the context of active intracellular signaling pathways in different cancers. SIRT3 is thus a novel target for preventing and treating cancer. In the present study, we explored the function of SIRT3 in non-small cell lung cancer (NSCLC) with the aim of elucidating the underlying mechanisms. We first determined the SIRT3 expression levels by real-time PCR, western blotting and immunohistochemistry on tissue microarrays of paired samples of NSCLC tissue and adjacent normal tissue from 70 patients with associated clinicopathological data. Levels of SIRT3 protein and mRNA were significantly increased in NSCLC tissue, compared with normal tissue $(\mathrm{P}<0.05)$. Expression of SIRT3 in NSCLC positively correlated with that of malignant biomarker Ki-67 $(\mathrm{P}<0.05)$ and oncogene $\mathrm{p}$-Akt $(\mathrm{P}<0.05)$. Patients with higher SIRT3 expression had a shorter overall survival duration $(\mathrm{P}<0.05)$. NSCLC tissue of squamous cell carcinoma type had higher SIRT3 expression compared with other types $(\mathrm{P}<0.05)$. Furthermore, among the clinicopathological variables examined, SIRT3 expression was correlated only with pathological type $(\mathrm{P}<0.05)$. In NSCLC
\end{abstract}

Correspondence to: Dr Yong Han, Department of Thoracic Surgery, Tangdu Hospital, Fourth Military Medical University, 1 Xinsi Road, Baqiao, Xi'an, Shaanxi 710038, P.R. China

E-mail: hanyong_td@163.com

Dr Lintao Jia, State Key Laboratory of Cancer Biology, Department of Biochemistry and Molecular Biology, Fourth Military Medical University, 169 Changle West Road, Xi'an, Shaanxi 710032, P.R. China E-mail: jialth@fmmu.edu.cn

*Contributed equally

Key words: SIRT3, Akt, non-small cell lung cancer, deacetylation cell lines, we found that downregulation of SIRT3 by siRNA decreased the activation of Akt, and that SIRT3 overexpression caused the activation of Akt. In addition, in a NSCLC cell line, SIRT3 was able to co-immunoprecipitate Akt and co-located with Akt, suggesting that SIRT3 regulates the activation of Akt through post-transcriptional modification. Our findings suggest that SIRT3 promotes the malignancy of NSCLC, showing an oncogenic preference towards squamous cell carcinoma, and that could represent a novel target for treatment.

\section{Introduction}

Lung cancer is one of the leading threats to human health globally. It is associated with high morbidity and mortality and a poor survival rate (1). Non-small cell lung cancer (NSCLC) accounts for approximately $85-90 \%$ of all lung cancers and compared with other types is relatively insensitive to treatment (2). Numerous studies have investigated the prevention and treatment of NSCLC; however, despite the gain in knowledge it remains a largely intractable disease (3). One promising treatment is targeted at the specific molecules borne by patients with mutations in oncogenes such as epidermal growth factor receptor (EGFR), V-Ki-ras2 Kirsten rat sarcoma viral oncogene homolog (KRAS) and anaplastic lymphoma kinase (ALK) (4). However, not all patients benefit and many exhibit tolerance. Hence, a search for novel molecular mechanisms involved in the etiology of NSCLC and its progression is required, with the ultimate aim of developing targeted therapy.

In recent years, proteomic studies demonstrated that more than 2000 proteins involved in diverse cellular processes could be acetylated, indicating that lysine acetylation is a promising target for cancer treatment (5-7). Deacetylases such as sirtuin-3 (SIRT3), a primary mitochondrial deacetylase, mediate a wide range of cellular processes involving acetylation (8). SIRT3 is involved in many diseases including diabetes, myocardial injury and cancer (9-12). However, SIRT3 functions as either an oncogene or an anti-oncogene in different cancers; the distinction is associated with complex biologic networks of signaling pathways depending upon the different genetic backgrounds in different cancer types (12-16). 
To date, there are still only few data available on the relationship between SIRT3 and NSCLC. In the present study, we investigated the relationship between SIRT3 and NSCLC in both clinical samples and cell lines to determine whether SIRT3 correlates with malignancy and examined the underlying mechanism.

\section{Materials and methods}

Patients and tissue specimens. NSCLC specimens accompanied by detailed clinical and pathological data were collected from 70 NSCLC patients who were diagnosed with NSCLC between April 2009 and January 2013 and underwent surgical resection at the Department of Thoracic Surgery, Tangdu Hospital, the Fourth Military Medical University (Xi'an, China). Cancerous tissue and adjacent normal lung tissue was obtained from each individual after resection of the tumor. All tissue specimens $(n=140)$ were snap-frozen in liquid nitrogen immediately after the collection for subsequent analysis. The cohort comprised $61(87.1 \%)$ males and $9(12.9 \%)$ females. The average age was 60 years (range, $31-79$ years). Mean survival time was 21.1 months. Tumor stage was defined according to the tumor-node-metastasis (TNM) classification of the American Joint Committee on Cancer (AJCC)/Union for International Cancer Control (UICC), the main tumor-staging system used in clinical practice and research.

Cell culture. NSCLC cell lines H520 and SW900 were purchased from the Cell Bank of the Chinese Academy of Sciences (Shanghai, China). Cells were cultured in RPMI-1640 medium supplemented with $10 \%$ fetal bovine serum (FBS), $100 \mathrm{mg} / 1$ penicillin, and $100 \mathrm{mg} / 1$ streptomycin at $37^{\circ} \mathrm{C}$ in a humidified $5 \% \mathrm{CO}_{2}$ atmosphere.

SIRT3 siRNA transfection. Briefly, SW900 and H520 cells were transfected with SIRT3 siRNA and control siRNA using Lipofectamine 2000 according to the manufacturer's instructions. SIRT3 siRNA1: 5'-CCAGCAUGAAAUACAUUUA TT-3', SIRT3 siRNA2: 5'-GCCCGACAUUGUGUUCUUU TT-3'; negative control siRNA: 5'-UUCUCCGAACGUGUC ACGUTT-3'.

Plasmid construction and cell transfection. SIRT3 full-length cDNA was synthesized from total RNA extracted from H520 cells using RT-PCR. Primers were designed as follows: forward, 5'-CCGCTCGAGATGGCGTTCTGGGGTTGG-3' and reverse, 5'-CGCGGATCCCTATTTGTCTGGTCCATCA AGC-3'.

The cDNA was then cloned into pcDNA3.1 (-) expression vector (Invitrogen, Carlsbad, CA, USA). The empty pcDNA3.1 (-) was used as a control. H520 cells were transfected with pcDNA3.1 (-)-SIRT3 or control empty pcDNA3.1 (-) using Lipofectamine 2000 according to the manufacturer's instructions.

Tissue microarray construction. The 140 specimens were fixed in $4 \%$ paraformaldehyde for $24 \mathrm{~h}$, dehydrated in a series of graded ethanol concentrations and then embedded in paraffin. According to hematoxylin and eosin-stained sections, representative areas were selected from which to construct a tissue microarray (TMA) using a tissue-arraying instrument (Quant Center Pannoramic MIDI). Each TMA contained 70 cancerous tissues and 70 corresponding adjacent normal tissues.

Immunohistochemistry. Paraformaldehyde-fixed and paraffin-embedded NSCLC TMA sections ( $4 \mu \mathrm{m}$ thick) were dewaxed in xylene and graded alcohols, hydrated and washed in phosphate-buffered saline (PBS). After pretreatment in a microwave oven, endogenous peroxidase was inhibited with $3 \%$ hydrogen peroxide for $25 \mathrm{~min}$. After blocking with $3 \%$ bovine serum albumin (BSA), slides were then incubated with the primary antibody (anti-SIRT3, sc-99143; anti-Ki-67, \#9449 Cell Signaling Technology; anti-Phospho-Akt Ser473, \#4060; Cell Signaling Technology) overnight in a moist chamber at $4^{\circ} \mathrm{C}$, washed in PBS and incubated with the appropriate horseradish peroxidase (HRP)-labeled goat anti-rabbit/mouse antibody. Slides were developed with liquid 3,'3-diaminobenzidine tetrahydrochloride (DAB) + Substrate Chromogen System (Dako) and counterstained with hematoxylin.

Immunofluorescence. Paraformaldehyde-fixed cells were incubated with primary antibody (SIRT3, sc-99143; Akt, 60203-1-Ig; Proteintech, Chicago, IL, USA) at $4^{\circ} \mathrm{C}$ overnight in a moist chamber, washed in PBS and incubated with Alexa Fluor 488 and CY3-conjugated secondary antibodies and 4',6-diamidino-2-phenylindole (DAPI) was used to stain the nucleus.

Immunohistochemistry evaluation. Semi-quantitative immunohistochemistry (IHC) detection was used to determine the protein levels, according to the intensity of staining and the percentage of positive cells. For antigens other than Ki-67, an $\mathrm{H}$-score was assigned, derived from $\mathrm{H}=\Sigma(\mathrm{pi}$ i $\mathrm{i})$, in which 'pi' represents the percentage of positive cells and ' $\mathrm{i}$ ' represents the intensity (17). For Ki-67, the index was defined as the percentage of $\mathrm{Ki}-67$-positive cells among all the cells of the TMA section. Slides were scanned and digitalized using a Pannoramic MIDI (3DHISTECH, Ltd., Budapest, Hungary) and analyzed using a Pannoramic Viewer v. 1.15.3 and NuclearQuant application for PV v.2.0.0.46136, both manufactured by 3DHISTECH (18).

Selection of cut-off score. The cut-off value used to differentiate SIRT3 expression into high and low levels was defined using a receiver operating characteristic (ROC) curve using the 0.1 criterion (19). The value closest to the point with both maximum sensitivity and specificity [i.e., the point $(0.0,0.1)$ on the curve] was selected as the cut-off value, leading to the largest number of tumors correctly classified as having or not having the clinical outcome $(20,21)$. High SIRT3 expressing samples were those with scores above the cut-off value, while the low samples had scores below or equal to the value.

Quantitative real-time PCR. Total RNA was extracted from the frozen tissue samples using RNAiso reagent (Takara Bio, Tokyo, Japan) according to the manufacturer's instructions, and then reverse transcribed to cDNA using PrimeScript RT Master Mix (Takara Bio). Levels of SIRT3 and $\beta$-actin were examined by real-time PCR (qPCR) using SYBR Premix Ex Taq II (Tli RNaseH Plus; Takara Bio) 
with a CFX96 Real-Time PCR detection system (Bio-Rad Laboratories, Hercules, CA, USA). Primers were designed as follows: SIRT3, forward, 5'-GCATCCCTGCCTCAAAGC-3' and reverse, 5'-GTCAGCCCGAATGTCCTC-3'; $\beta$-actin, forward, 5'-GATCATTGCTCCTCCTGAGC-3' and reverse, 5'-ACTCCTGCTTGCTGATCCAC-3'. Conditions were as follows: one cycle of $95^{\circ} \mathrm{C}$ for $3 \mathrm{~min}$, followed by 45 amplification cycles at $95^{\circ} \mathrm{C}$ for $10 \mathrm{sec}$, and annealing and elongation at $55^{\circ} \mathrm{C}$ for $30 \mathrm{sec}$. The relative expression of SIRT3 was normalized to endogenous $\beta$-actin using the comparative threshold cycle $\left(2^{-\Delta \mathrm{CT}}\right)$ method. Similarly, the relative expression of SIRT3 was normalized to the control groups using the comparative threshold cycle $\left(2^{-\Delta \Delta \mathrm{CT}}\right)$ method.

Western blotting. Cell lysates were prepared from the frozen tissue samples using radio immunoprecipitation (RIPA) lysis buffer combined with a phosphatase and protease inhibitor cocktail. Lysates were boiled with sodium dodecyl sulfate(SDS) loading buffer and then separated by SDS-polyacrylamide gel electrophoresis (PAGE). Proteins were transferred to a nitrocellulose membrane, which was later incubated with a primary specific antibody (anti-SIRT3, \#2627; Cell Signaling Technology; anti-pan Akt, \#4691; Cell Signaling Technology; anti-Phospho-Akt Ser473, \#4060; Cell Signaling Technology; anti- $\beta$-actin: \#3700; Cell Signaling Technology), all purchased in 5\% non-fat milk, followed by HRP-conjugated anti-rabbit/ mouse secondary antibody. ECL detection reagent (Merck Millipore, Darmstadt, Germany) was used to demonstrate the protein bands.

Immunoprecipitation. Primary antibody was added to magnetic beads diluted in PBS with Tween-20, and then incubated with rotation at room temperature to allow crosslinking. After centrifugation, the supernatant was removed and the beads-antibody complex was washed with the aid of a magnet. Cell samples extracted through NP40 cell lysis buffer with phosphatase and protease inhibitor cocktail were added to the beads-antibody complex to immunoprecipitate the target antigen. Finally, after removing the supernatant, and washing the beads-antibody-antigen complex, SDS loading buffer was added for examination on a gel. Conformation-specific mouse anti-rabbit HRP-conjugated anti-rabbit/mouse secondary antibody (\#5127; Cell Signaling Technology) was used to avoid $\mathrm{IgG}$ subunit interference.

Statistical analysis. Statistical analyses were performed using SPSS 16.0 software (SPSS, Inc., Chicago, IL, USA). ROC curve analysis was applied to define the cut-off value for high expression of SIRT3 using the 0.1 criterion, and the area under the curve (AUC) was measured. A Wilcoxon matched paired test, paired t-test, one-way ANOVA analysis and SNK-q test were used to compare the SIRT3 expression pattern according to numeric data types. Pearson correlation analysis was used to estimate the relationship between SIRT3 expression and other proteins. A $\chi^{2}$ test was performed to analyze the correlation between SIRT3 expression and clinicopathological parameters. Overall survival (OS) was defined as the time from initial diagnosis to death and assessed using the Kaplan-Meier method and compared by the log-rank test. $\mathrm{P}=0.05$ (two-tailed) was considered statistically significant.
Table I. Correlation between SIRT3 expression and clinical variables of the NSCLC cases.

\begin{tabular}{|c|c|c|c|c|}
\hline \multirow[b]{2}{*}{ Variables } & \multirow[b]{2}{*}{$\begin{array}{l}\text { Cases } \\
(n=70)\end{array}$} & \multicolumn{2}{|c|}{$\begin{array}{c}\text { SIRT3 } \\
\text { expression }\end{array}$} & \multirow[b]{2}{*}{$\begin{array}{l}\text { P-value } \\
\left(\chi^{2} \text { test }\right)\end{array}$} \\
\hline & & $\begin{array}{l}\text { High } \\
(n=42)\end{array}$ & $\begin{array}{c}\text { Low } \\
(n=28)\end{array}$ & \\
\hline Age (years) & & & & 0.052 \\
\hline$>60$ & 37 & 18 & 19 & \\
\hline$\leq 60$ & 33 & 24 & 9 & \\
\hline Gender & & & & 0.732 \\
\hline Male & 61 & 36 & 25 & \\
\hline Female & 9 & 6 & 3 & \\
\hline Tumor location & & & & 1 \\
\hline Left lung & 30 & 18 & 12 & \\
\hline Right lung & 40 & 24 & 16 & \\
\hline Tumor size $(\mathrm{cm})$ & & & & 0.215 \\
\hline$>5$ & 44 & 29 & 15 & \\
\hline$\leq 5$ & 26 & 13 & 13 & \\
\hline \multicolumn{5}{|l|}{ Lymphatic invasion } \\
\hline Yes & 44 & 24 & 20 & 0.313 \\
\hline No & 26 & 18 & 8 & \\
\hline \multicolumn{5}{|l|}{ Tumor stage } \\
\hline $\mathrm{T} 1 / \mathrm{T} 2$ & 27 & 13 & 14 & 0.136 \\
\hline $\mathrm{T} 3 / \mathrm{T} 4$ & 43 & 29 & 14 & \\
\hline Clinical stage & & & & 0.467 \\
\hline Stage II & 29 & 19 & 10 & \\
\hline Stage III & 41 & 23 & 18 & \\
\hline Pathology type & & & & 0.001 \\
\hline Adenocarcinoma & 13 & 5 & 8 & \\
\hline Squamous carcinoma & 47 & 35 & 12 & \\
\hline Adenosquamous carcinoma & 10 & 2 & 8 & \\
\hline Therapeutic method & & & & 0.609 \\
\hline Surgery + chemotherapy & 47 & 27 & 20 & \\
\hline Surgery & 23 & 15 & 8 & \\
\hline
\end{tabular}

\section{Results}

The expression of SIRT3 is upregulated in human NSCLC tissue. To investigate the expression pattern of SIRT3 in NSCLC, we first examined the protein level in 70 paired clinical samples of NSCLC and adjacent normal lung tissue by IHC. According to the results of the TMA-based IHC, SIRT3 was noticeably upregulated in the cancerous tissue, compared with the normal tissues. A paired t-test showed that there was a significant difference between the cancerous and normal sample groups $(\mathrm{P}=0.004$; Fig. 1A). A representative pair of IHC-stained tissues and five paired western blotting results demonstrating the aberrant upregulated SIRT3 expression in NSCLC are shown in Fig. 1B. Next, we investigated SIRT3 mRNA expression levels in 16 paired tissue samples. SIRT3 mRNA levels were markedly higher in the cancerous, 

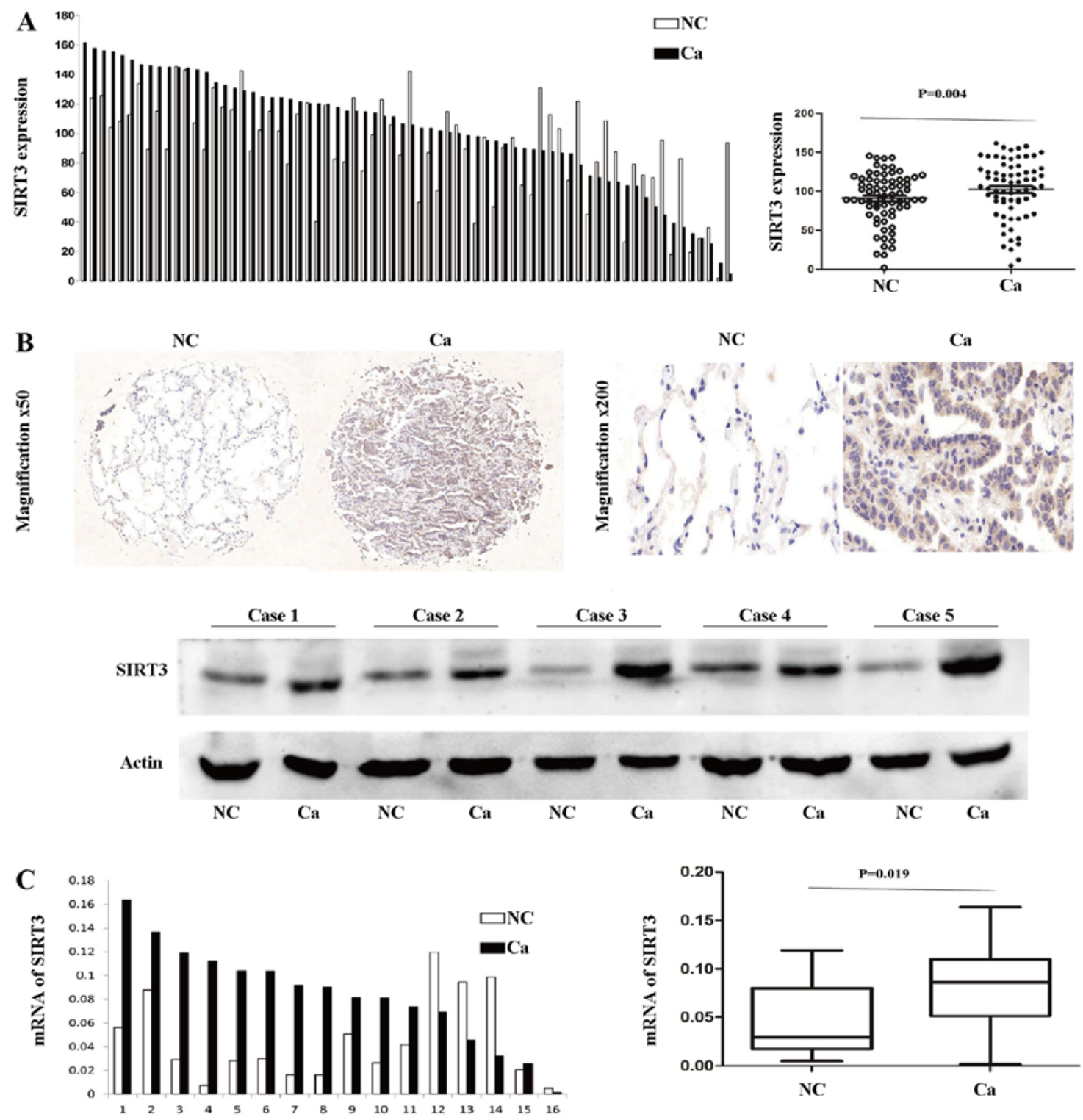

D
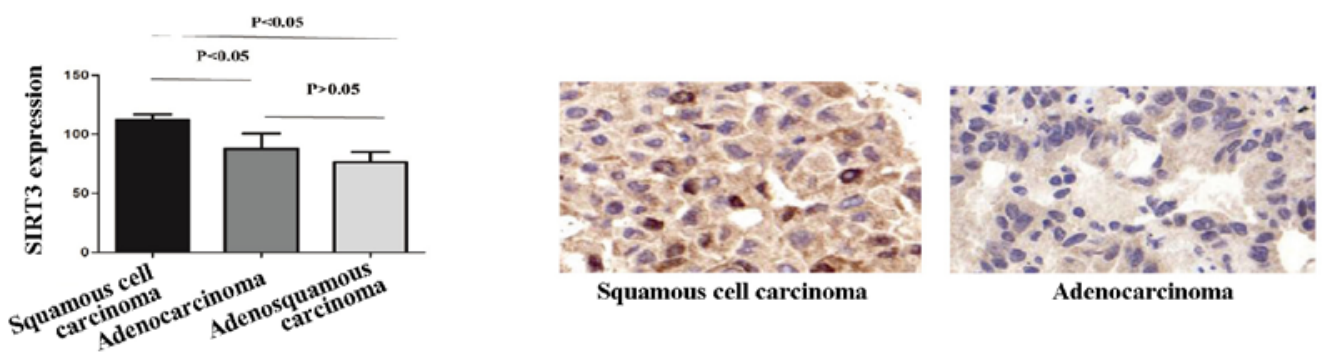

Figure 1. Expression of SIRT3 in human NSCLC cancerous tissues compared with normal lung tissues (NC, normal lung tissue as control; Ca, non-small cell lung cancer tissue). (A) Protein levels of SIRT3 in 70 pairs of cancerous tissues and adjacent normal tissues according to IHC scores. Paired t-test, $\mathrm{P}=0.004$. (B) One paired representative example of SIRT3 IHC from one NSCLC patient showing higher SIRT3 expression in the cancerous tissue. Magnification, x50 (left-hand images). Magnification, x200 (right-hand images). Western blotting results from five paired cancerous and normal tissues showing aberrant SIRT3 protein upregulation in the cancerous tissue. (C) Normalized SIRT3 mRNA levels in 16 pairs of cancerous tissues and adjacent normal tissues by qPCR. Wilcoxon matched paired test, $\mathrm{P}=0.019$. (D) SNK-q test to determine the difference in SIRT3 expression between different NSCLC types ( $<<0.05$; left), Representative examples of SIRT3 IHC showing higher expression in squamous cell carcinoma (magnification, x200).

compared with the normal lung, tissue group (Wilcoxon matched paired test, $\mathrm{P}=0.019$; Fig. 1C). In addition, SIRT3 was more upregulated in squamous cell carcinoma type NSCLC (Fig. 1D).

Taken together, these data demonstrate that SIRT3 expression is upregulated in human NSCLC cancerous tissue compared with normal lung tissue, and to an even greater extent in squamous cell carcinoma.

SIRT3 expression positively correlates with the malignant biomarker Ki-67 and oncogene p-Akt in NSCLC. Next, we investigated the inter-relationship between SIRT3 expression 

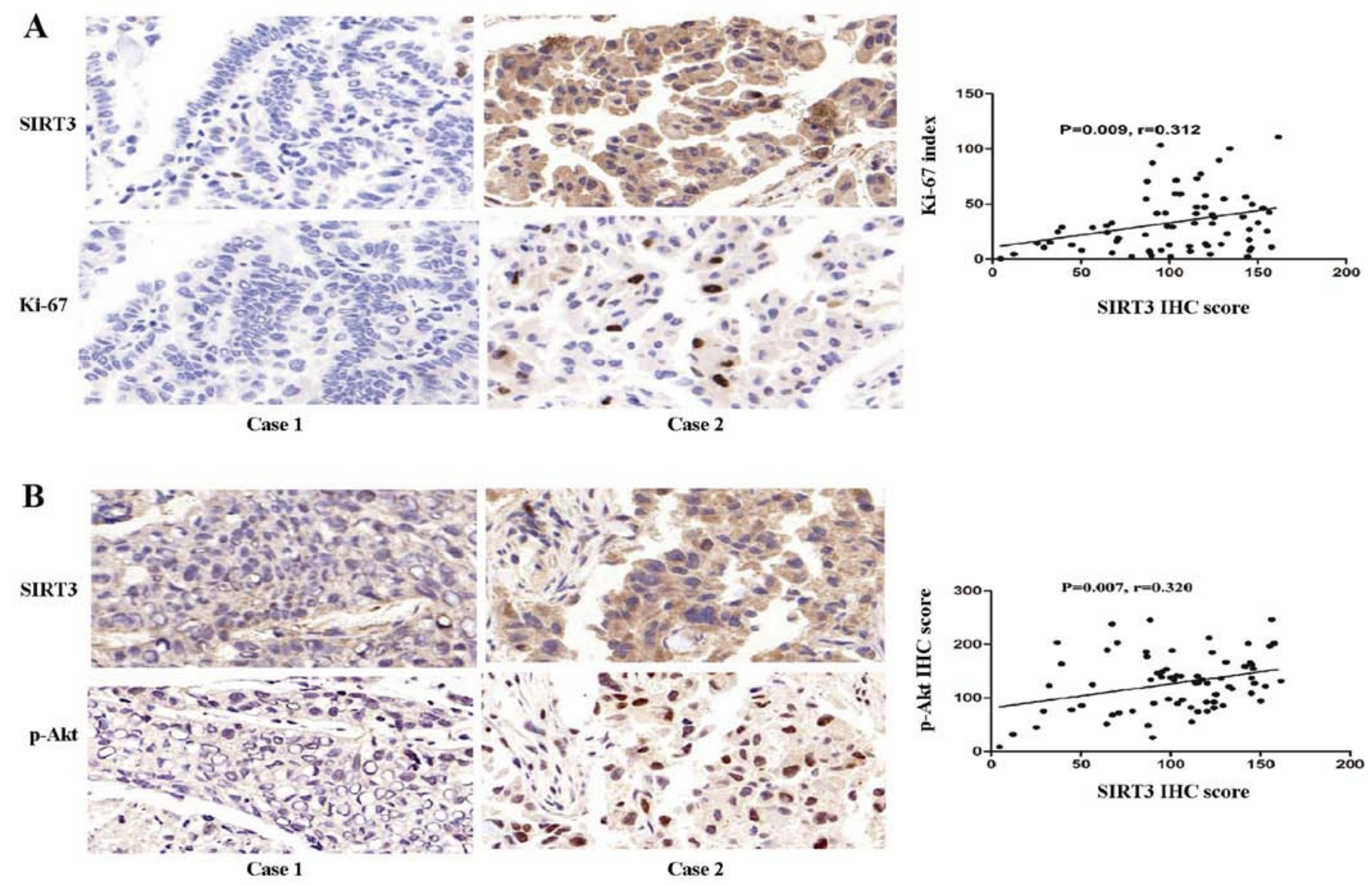

Figure 2. Correlation of SIRT3 expression with malignant proliferative biomarkers in NSCLC. (A) Two paired representative examples of SIRT3 and Ki-67 IHC showing that SIRT3 positively correlates with Ki-67 in NSCLC. Magnification, x200. Pearson correlation analysis demonstrated a positive correlation between SIRT3 and Ki-67. r, coefficient of correlation. P=0.009. (B) Two paired representative examples of SIRT3 and p-Akt IHC showing that SIRT3 positively correlates with the activation status of Akt in NSCLC. Magnification, x200. Pearson correlation analysis demonstrates positive correlation between SIRT3 and p-Akt IHC scores. r, coefficient of correlation. P=0.007.

and $\mathrm{Ki}-67$, a biomarker of malignant proliferation and the oncogene p-Akt, in NSCLC. The results showed that SIRT3 expression was positively correlated with $\mathrm{Ki}-67$ expression $(\mathrm{P}=0.009$, coefficient of correlation $(\mathrm{r})=0.312$; Fig. 2A). SIRT3 expression was also positively correlated with p-Akt (ser473) $(\mathrm{P}=0.007$, r=0.320; Fig. 2B).

High SIRT3 expression indicates a shorter OS. To determine the significance of SIRT3 expression on prognosis in NSCLC patients, we defined an optimal cut-off value for high or low SIRT3 expression using ROC curve analysis. The results demonstrated that divided cut-off value for survival status has the shortest distance from the curve to the point $(0.0$, 1.0) $(\mathrm{P}=0.019$; Fig. 3A). We then carried out a retrospective analysis of the outcomes of the 70 study patients. The median survival time was 14.97 months for the patients with high SIRT3 expression, while it was 19.29 months for patients with a low level of SIRT3. Kaplan-Meier survival analyses showed that patients with high SIRT3 expression had a shorter OS duration ( $\mathrm{P}=0.028$; Fig. 3B).

No correlation was found between the SIRT3 expression and the clinicopathological variables of NSCLC except cancer types. To estimate the clinical significance of SIRT3 expression in NSCLC, we analyzed the association between the SIRT3 expression and the clinicopathological characteristics. Correlations were observed between the SIRT3 expression and the pathological type $(\mathrm{P}=0.001)$. There were no statistical connections between the SIRT3 expression and the other clinicopathological parameters, such as age, gender, tumor location, clinical stage and therapeutic method $(\mathrm{P}>0.05$; Table I).

SIRT3 promotes the activation of the Akt signaling pathway in NSCLC. To explore the mechanism underlying the involvement of SIRT3 in NSCLC, we first generated two NSCLC cell lines (H520 and SW900) with downregulated SIRT3 using RNA interference. We found that, in both SIRT3 downregulated cell lines, expression of p-Akt (ser473), a surrogate marker of Akt activity, was decreased, while expression of total Akt showed no significant difference (Fig. 4A). We then generated SIRT3 overexpressing H520 cells using the pcDNA3.1 expression vector and found that SIRT3 overexpression caused the activation of Akt (Fig. 4B). Lastly, we demonstrated that SIRT3 could co-precipitate and was co-located with Akt and vice versa in endogenous $\mathrm{H} 520$ cell state, indicating that SIRT3 may mediate activation of Akt by their binding or function in a protein complex (Fig. 4C and D). These results indicate that SIRT3 promotes activation of Akt signaling pathways in NSCLC. 

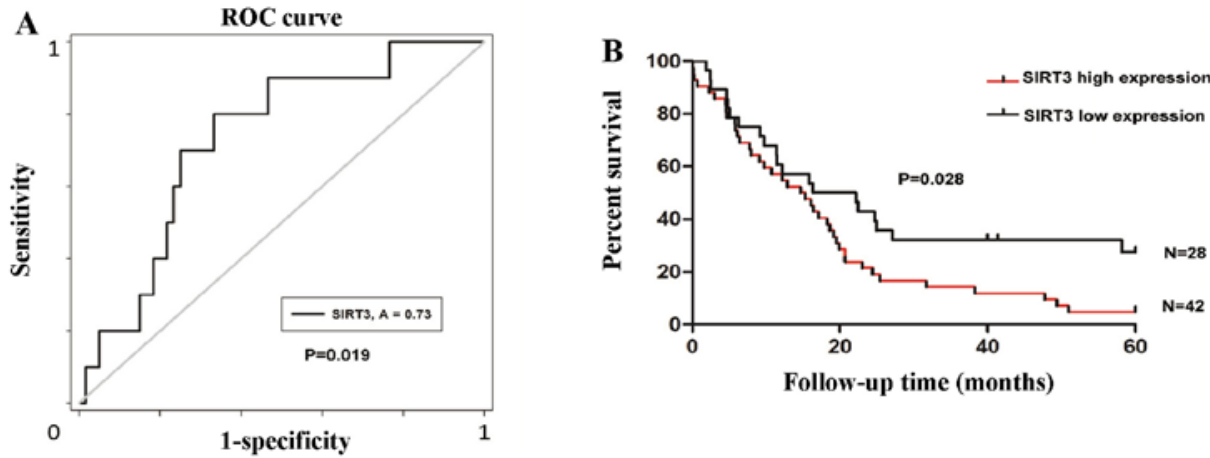

Figure 3. SIRT3 expression and OS of NSCLC patients. (A) ROC curve analysis to determine the optimal cut-off value for separating high and low level SIRT3 expression. A, area under the curve. $\mathrm{P}=0.019$. (B) Kaplan-Meier survival estimate according to cancer SIRT3 expression. $\mathrm{P}=0.028$.

A

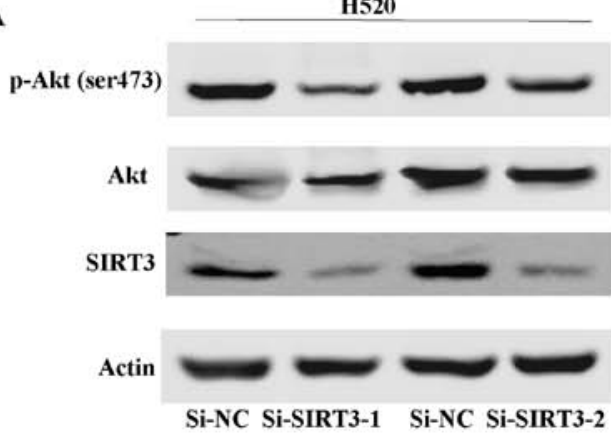

B

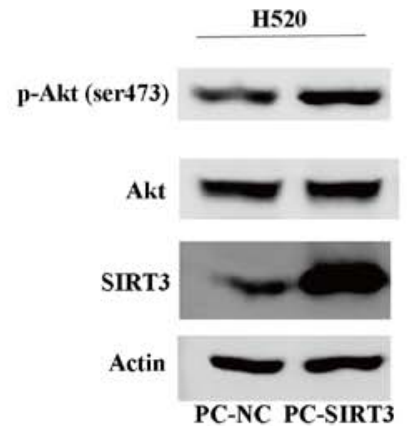

D

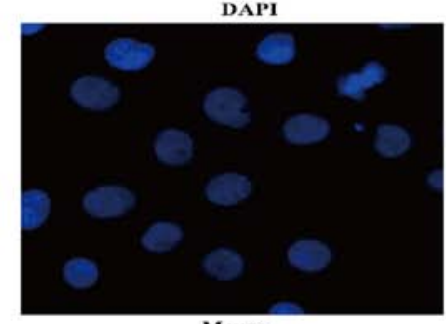

Merge

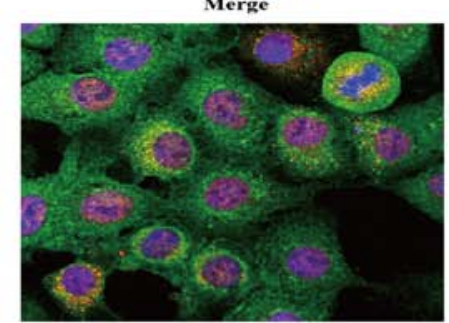

SW900

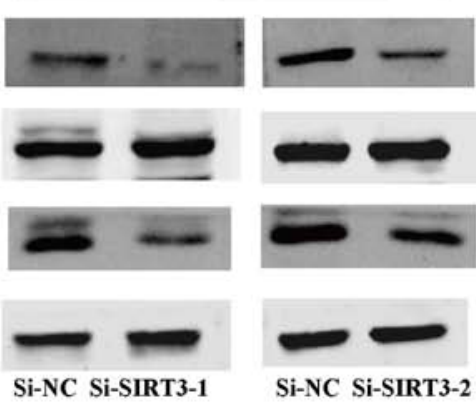

Si-NC Si-SIRT3-2

C

WB $10 \%$ input $\quad$ Ig G $\quad$ SIRT3 Akt

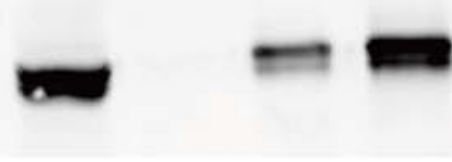

SIRT3

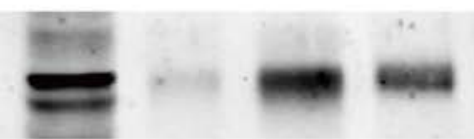

SIRT3

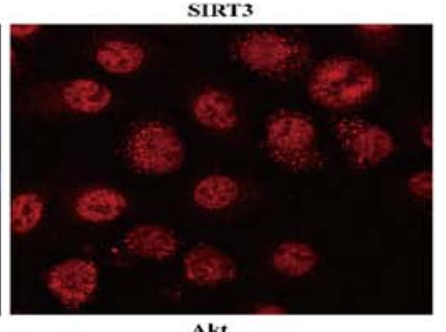

Akt

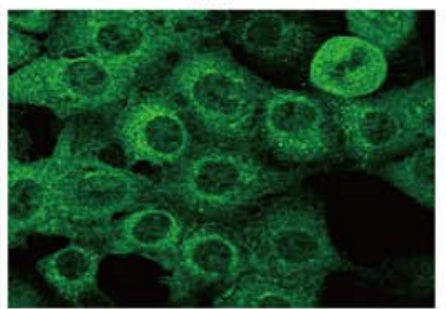

Figure 4. SIRT3-mediated activation of Akt in NSCLC cell lines. (A) SIRT3 knockdown: Protein expression of Akt, p-Akt and SIRT3 by western blotting of H520 and SW900 NSCLC cell lines transfected with scrambled si-RNA negative control (Si-NC) or Si-SIRT3. (B) SIRT3 overexpression: Protein expression of Akt, p-Akt and SIRT3 by western blotting of H520 NSCLC cell lines transfected with empty pcDNA3.1 (-) negative control (PC-NC) or PC-SIRT3. (C) Co-precipitation of SIRT3 and Akt: Anti-SIRT3 antibody immunoprecipitation (IP) of endogenous Akt and vice versa, in the H520 cell line. (D) Co-localization of SIRT3 and Akt: H520 NSCLC cell line stained with anti-Akt antibody/Alexa Fluor 488 (green) and anti-SIRT3 antibody/CY3 (red), and DAPI nuclear stain (blue). 


\section{Discussion}

We propose that SIRT3 has oncogenic activity in NSCLC, and that the underlying molecular mechanism is associated with activation of Akt signaling pathways. The present study showed the oncogene function of SIRT3 in NSCLC, and it suggests it is most strongly associated with squamous cell carcinoma. We demonstrated that SIRT3 can activate the Akt signaling pathway and that this interaction may involve posttranscriptional acetylation modification of SIRT3. However, the study was limited, as currently we cannot account for the possible involvement of SIRT3 in other signaling networks that initiate and promote the progress of NSCLC. Further systematic research will be necessary to elucidate the specific pathologic activity of SIRT3.

In recent years, numerous studies have indicated ambiguity in the function of SIRT3 in different cancer types (13-15). For example, in breast cancer, SIRT3 suppresses proliferation and anaerobic glycolysis, and increases apoptosis via the depletion of reactive oxygen species (ROS) $(22,23)$. Furthermore, in some cancers SIRT3 appears to act as an anti-oncogene by delaying the degradation of p53 $(24,25)$. By contrast, in some cancer types, such as esophageal cancer and oral squamous cell carcinoma, SIRT3 is considered to promote carcinogenesis $(26,27)$. However, with respect to NSCLC, according to the small amount of research available in the literature, there is no consensus on SIRT3 function, and while it has apparent clinical significance, data are insufficient to explain the underlying molecular mechanisms $(25,28)$. In addition, regarding the relationship between Akt and SIRT3, previous reports propose that SIRT3 could inhibit the activation of Akt by preventing ROS-mediated Ras-PI3K-Akt activation (29-31). However, as well as ROS-mediated activation of Akt, other recent research points to post-translational modifications like acetylation being another significant means of regulating this central node of activity, where acetylation modification of Akt or its critical activator PDK1 could enhance their membrane transport and interaction with each other $(32,33)$. This breakthrough in finding a mediation mechanism for oncogenes prompted us to explore the deacetylation function of SIRT3 upon critical proteins in malignant transformation.

In the present study, we demonstrated that SIRT3 expression was significantly increased in cancerous tissues compared with normal lung tissues at both the transcription and protein level, which suggested that SIRT3 had a significant possibility of being involved in promoting the malignancy of NSCLC. Our finding that SIRT3 expression was positively correlated with established malignant biomarkers of NSCLC, Ki-67 (a specific cellular biomarker of proliferation) (34) and p-Akt (representing the activation status of oncogene Akt), strongly suggests that SIRT3 is also positively correlated with the malignancy of NSCLC. Furthermore, we showed that patients with high expression of SIRT3 have a poor prognosis, indicating that SIRT3 has prognostic clinical significance. In addition, we also found that the only clinicopathological variable with which high SIRT3 expression significantly correlated was pathologic type, namely, squamous cell carcinoma. This finding is consistent with previous studies that demonstrated that SIRT3 can play conflicting roles in cancer because there are distinct types. Akt signaling pathways are classical onco- genic pathways in numerous cancers via diverse mechanisms $(35,36)$; hence the finding that SIRT3 was involved in the pathway further implicates it in the malignancy of NSCLC. We showed that overexpression of SIRT3 enhanced the activation of Akt, and our co-precipitation and co-location studies suggested that SIRT3 was deacetylating Akt, in a similar role to that known for other SIRTs that deacetylate and activate Akt or an upstream mediator like PDK1 $(31,37)$.

These results provided clues to understand the mechanisms underlying the malignant function of SIRT3 in NSCLC and simultaneously disclosed a novel relationship between SIRT3 and Akt, namely, that SIRT3 could function as an activator upstream of the Akt signaling pathway but not via the blocking of ROS-mediated Ras-PI3K-Akt activation (29-31). How SIRT3 regulates Akt now requires further investigation; it could be via lysine deacetylation of Akt itself or of numerous known substrates of SIRT3, containing some classical or novel regulators of the Akt signaling pathway.

The present study also revealed clues to help interpret the conflicting role of SIRT3 in cancer by comparing the genetic background of squamous cell with other types of carcinoma. Previous studies show that, in squamous cell carcinomas, such as esophageal cancer and oral squamous cell carcinoma, SIRT3 behaves as an oncogene but, in cancers composed mainly of adenocarcinoma, like breast cancer, SIRT3 acts as an anti-oncogene. However, ascribing these distinctions is likely to be oversimplifying as the role of SIRT3 in other cancers, like melanoma and renal cell cancer, is contradictory $(38,39)$. We surmise that this phenomenon can be attributed to different types of 'oncogene addiction' at play in different cancers depending upon the molecular pathways involved $(40,41)$, thereby allowing SIRT3 to play variable roles. Unraveling this complex interplay is likely to provide novel cancer therapeutic targets and remains an important scientific challenge.

In summary, our studies indicate that SIRT3 could be applied as a novel target for investigating NSCLC, helping us to gain a better understanding of the initiation and progression of NSCLC. We now plan to investigate SIRT3 function in NSCLC in more specific and comprehensive ways and to further elucidate the relationship between SIRT3 and NSCLC.

\section{Acknowledgements}

We would like to thank all the members of the Department of Thoracic Surgery, Tangdu Hospital, Fourth Military Medical University, Xi'an, China and of the State Key Laboratory of Cancer Biology, Department of Biochemistry and Molecular Biology, Fourth Military Medical University, Xi'an, China for providing the clinically annotated samples and fine experimental conditions. The present study was supported by funds from the Special Clinical Research Fund from the Wu JiePing Medical Foundation (320.6750.15095), and the Natural Science and Technology Foundation Research Key Project of Shaanxi Province (2015JZ 024), China.

\section{References}

1. Jemal A, Bray F, Center MM, Ferlay J, Ward E and Forman D: Global cancer statistics. CA Cancer J Clin 61: 69-90, 2011. 
2. Molina JR, Yang P, Cassivi SD, Schild SE and Adjei AA Non-small cell lung cancer: Epidemiology, risk factors, treatment, and survivorship. Mayo Clin Proc 83: 584-594, 2008.

3. Goldstraw P, Ball D, Jett JR, Le Chevalier T, Lim E, Nicholson AG and Shepherd FA: Non-small-cell lung cancer. Lancet 378 : $1727-1740,2011$.

4. Pao W and Girard N: New driver mutations in non-small-cell lung cancer. Lancet Oncol 12: 175-180, 2011.

5. Choudhary C, Kumar C, Gnad F, Nielsen ML, Rehman M, Walther TC, Olsen JV and Mann M: Lysine acetylation targets protein complexes and co-regulates major cellular functions. Science 325: 834-840, 2009.

6. Kim SC, Sprung R, Chen Y, Xu Y, Ball H, Pei J, Cheng T, Kho Y, Xiao H, Xiao L, et al: Substrate and functional diversity of lysine acetylation revealed by a proteomics survey. Mol Cell 23 607-618, 2006

7. Guan KL and Xiong Y: Regulation of intermediary metabolism by protein acetylation. Trends Biochem Sci 36: 108-116, 2011.

8. Ahn BH, Kim HS, Song S, Lee IH, Liu J, Vassilopoulos A, Deng CX and Finkel T: A role for the mitochondrial deacetylase Sirt3 in regulating energy homeostasis. Proc Natl Acad Sci USA 105: 14447-14452, 2008.

9. Jing E, Emanuelli B, Hirschey MD, Boucher J, Lee KY, Lombard D, Verdin EM and Kahn CR: Sirtuin-3 (Sirt3) regulates skeletal muscle metabolism and insulin signaling via altered mitochondrial oxidation and reactive oxygen species production. Proc Natl Acad Sci USA 108: 14608-14613, 2011.

10. Sundaresan NR, Samant SA, Pillai VB, Rajamohan SB and Gupta MP: SIRT3 is a stress-responsive deacetylase in cardiomyocytes that protects cells from stress-mediated cell death by deacetylation of Ku70. Mol Cell Biol 28: 6384-6401, 2008.

11. Kumar S and Lombard DB: Mitochondrial sirtuins and their relationships with metabolic disease and cancer. Antioxid Redox Signal 22: 1060-1077, 2015

12. Haigis MC, Deng CX, Finley LWS, Kim HS and Gius D: SIRT3 is a mitochondrial tumor suppressor: A scientific tale that connects aberrant cellular ROS, the Warburg effect, and carcinogenesis Cancer Res 72: 2468-2472, 2012.

13. Finley LWS and Haigis MC: Metabolic regulation by SIRT3: Implications for tumorigenesis. Trends Mol Med 18: 516-523, 2012.

14. Alhazzazi TY, Kamarajan P, Verdin E and Kapila YL: Sirtuin-3 (SIRT3) and the hallmarks of cancer. Genes Cancer 4: 164-171, 2013.

15. Chen Y, Fu LL, Wen X, Wang XY, Liu J, Cheng Y and Huang J: Sirtuin-3 (SIRT3), a therapeutic target with oncogenic and tumorsuppressive function in cancer. Cell Death Dis 5: e1047, 2014.

16. Xiong Y, Wang M, Zhao J, Han Y and Jia L: Sirtuin 3: A Janus face in cancer (Review). Int J Oncol 49: 2227-2235, 2016.

17. Budwit-Novotny DA, McCarty KS, Cox EB, Soper JT, Mutch DG, Creasman WT, Flowers JL and McCarty KS Jr: Immunohistochemical analyses of estrogen receptor in endometrial adenocarcinoma using a monoclonal antibody. Cancer Res 46: 5419-5425, 1986.

18. Shin SJ, Roh J, Cha HJ, Choi YD, Kim JM, Min SK, Kim JE Eom DW, Lee H, Kim HJ, et al: TCL1 expression predicts overall survival in patients with mantle cell lymphoma. Eur J Haematol 95: 583-594, 2015

19. DeLong ER, DeLong DM and Clarke-Pearson DL: Comparing the areas under two or more correlated receiver operating characteristic curves: A nonparametric approach. Biometrics 44: 837-845, 1988.

20. Cai MY, Zhang B, He WP, Yang GF, Rao HL, Rao ZY, Wu QL, Guan XY, Kung HF, Zeng YX, et al: Decreased expression of PinX1 protein is correlated with tumor development and is a new independent poor prognostic factor in ovarian carcinoma. Cancer Sci 101: 1543-1549, 2010

21. Zlobec I, Steele R, Terracciano L, Jass JR and Lugli A: Selecting immunohistochemical cut-off scores for novel biomarkers of progression and survival in colorectal cancer. J Clin Pathol 60 $1112-1116,2007$.
22. Bell EL, Emerling BM, Ricoult SJH and Guarente L: SirT3 suppresses hypoxia inducible factor $1 \alpha$ and tumor growth by inhibiting mitochondrial ROS production. Oncogene 30: 2986-2996, 2011.

23. Wei L, Zhou Y, Qiao C, Ni T, Li Z, You Q, Guo Q and Lu N Oroxylin A inhibits glycolysis-dependent proliferation of human breast cancer via promoting SIRT3-mediated SOD2 transcription and HIF1 $\alpha$ destabilization. Cell Death Dis 6: e1714, 2015.

24. Zhao K, Zhou Y, Qiao C, Ni T, Li Z, Wang X, Guo Q, Lu N and Wei L: Oroxylin A promotes PTEN-mediated negative regulation of MDM2 transcription via SIRT3-mediated deacetylation to stabilize p53 and inhibit glycolysis in wt-p53 cancer cells. J Hematol Oncol 8: 41, 2015.

25. Xiao K, Jiang J, Wang W, Cao S, Zhu L, Zeng H, Ouyang R, Zhou R and Chen P: Sirt3 is a tumor suppressor in lung adenocarcinoma cells. Oncol Rep 30: 1323-1328, 2013.

26. Zhao Y, Yang H, Wang X, Zhang R, Wang C and Guo Z: Sirtuin-3 (SIRT3) expression is associated with overall survival in esophageal cancer. Ann Diagn Pathol 17: 483-485, 2013.

27. Alhazzazi TY, Kamarajan P, Joo N, Huang JY, Verdin E, D'Silva NJ and Kapila YL: Sirtuin-3 (SIRT3), a novel potential therapeutic target for oral cancer. Cancer 117: 1670-1678, 2011.

28. Li H, Feng Z, Wu W, Li J, Zhang J and Xia T: SIRT3 regulates cell proliferation and apoptosis related to energy metabolism in non-small cell lung cancer cells through deacetylation of NMNAT2. Int J Oncol 43: 1420-1430, 2013.

29. Zhang YY and Zhou LM: Sirt3 inhibits hepatocellular carcinoma cell growth through reducing Mdm2-mediated p53 degradation. Biochem Biophys Res Commun 423: 26-31, 2012.

30. Quan Y, Wang N, Chen Q, Xu J, Cheng W, Di M, Xia W and Gao WQ: SIRT3 inhibits prostate cancer by destabilizing oncoprotein $\mathrm{c}-\mathrm{MYC}$ through regulation of the PI3K/Akt pathway. Oncotarget 6: 26494-26507, 2015.

31. Pillai VB, Sundaresan NR and Gupta MP: Regulation of Akt signaling by sirtuins: Its implication in cardiac hypertrophy and aging. Circ Res 114: 368-378, 2014.

32. Chan CH, Jo U, Kohrman A, Rezaeian AH, Chou PC, Logothetis C and Lin HK: Posttranslational regulation of Akt in human cancer. Cell Biosci 4: 59, 2014.

33. Risso G, Blaustein M, Pozzi B, Mammi P and Srebrow A: Akt/ PKB: One kinase, many modifications. Biochem J 468: 203-214, 2015.

34. Scholzen T and Gerdes J: The Ki-67 protein: From the known and the unknown. J Cell Physiol 182: 311-322, 2000.

35. Bauer TM, Patel MR and Infante JR: Targeting PI3 kinase in cancer. Pharmacol Ther 146: 53-60, 2015.

36. Testa JR and Bellacosa A: AKT plays a central role in tumorigenesis. Proc Natl Acad Sci USA 98: 10983-10985, 2001.

37. Sundaresan NR, Pillai VB, Wolfgeher D, Samant S, Vasudevan $P$, Parekh V, Raghuraman H, Cunningham JM, Gupta M and Gupta MP: The deacetylase SIRT1 promotes membrane localization and activation of Akt and PDK1 during tumorigenesis and cardiac hypertrophy. Sci Signal 4: ra46, 2011.

38. George J, Nihal M, Singh CK, Zhong W, Liu X and Ahmad N: Pro-proliferative function of mitochondrial sirtuin deacetylase SIRT3 in human melanoma. J Invest Dermatol 136: 809-818, 2016.

39. Choi J, Koh E, Lee YS, Lee HW, Kang HG, Yoon YE, Han WK, Choi KH and Kim KS: Mitochondrial Sirt3 supports cell proliferation by regulating glutamine-dependent oxidation in renal cell carcinoma. Biochem Biophys Res Commun 474: 547-553, 2016.

40. Weinstein IB: Cancer. Addiction to oncogenes - the Achilles heal of cancer. Science 297: 63-64, 2002.

41. Fisher GH, Wellen SL, Klimstra D, Lenczowski JM, Tichelaar JW, Lizak MJ, Whitsett JA, Koretsky A and Varmus HE: Induction and apoptotic regression of lung adenocarcinomas by regulation of a $K$-Ras transgene in the presence and absence of tumor suppressor genes. Genes Dev 15: 3249-3262, 2001. 